\title{
Transatlantica
}

Revue d'études américaines. American Studies Journal

Révolution

\section{Thomas Paine et l'éloge des révolutions}

\section{Nathalie Caron}

\section{(2) OpenEdition}

Journals

Édition électronique

URL : http://journals.openedition.org/transatlantica/1145

DOI : 10.4000/transatlantica.1145

ISSN : 1765-2766

Éditeur

AFEA

\section{Référence électronique}

Nathalie Caron, «Thomas Paine et l'éloge des révolutions », Transatlantica [En ligne], 2 | 2006, mis en ligne le 07 juillet 2006, consulté le 29 avril 2021. URL : http://journals.openedition.org/transatlantica/ 1145 ; DOI : https://doi.org/10.4000/transatlantica.1145

Ce document a été généré automatiquement le 29 avril 2021.

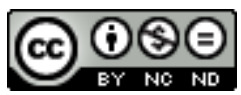

Transatlantica - Revue d'études américaines est mis à disposition selon les termes de la licence Creative Commons Attribution - Pas d'Utilisation Commerciale - Pas de Modification 4.0 International. 


\title{
Thomas Paine et l'éloge des révolutions
}

\author{
Nathalie Caron
}

Un homme engagé

1 Thomas Paine (1737-1809), républicain déiste, est l'un de ces hommes des Lumières qui ont le mieux illustré, par leurs écrits, par leurs engagements politiques et religieux, mais aussi par leur existence même, leurs déplacements géographiques et leurs activités, les révolutions transatlantiques du XVIII ${ }^{\mathrm{e}}$ siècle. Ce "citoyen du monde ", ou « citoyen universel » comme il aimait se définir, successivement anglais, américain et français, fut, tout au long de sa vie, l'un des défenseurs les plus ardents et les plus généreux du processus révolutionnaire ${ }^{1}$. Pour un homme tel que Paine, seuls l'usage bien compris de la raison et la révolution «active" pouvaient, en permettant l'épanouissement de l'individu rendu enfin à lui-même, assurer le progrès du genre humain et poser les conditions du bonheur individuel et collectif (Les droits de l'homme 266) $)^{2}$.

2 Paine incarne parfaitement bien le transatlantisme, voire le cosmopolitisme des révolutions $\mathrm{du} \mathrm{XVIII}^{\mathrm{e}}$ siècle. Il se prononça en effet avec ferveur pour la révolution américaine comme pour la révolution française, deux révolutions auxquelles il participa activement. Et toute sa vie, de façon presque désespérée, il nourrit l'espoir d'une révolution en Angleterre, son pays natal. Il fit son possible - il comptait beaucoup sur Napoléon - pour qu'y éclate, cette fois-là, une véritable révolution, la révolution dite glorieuse de 1689 n'étant pour lui qu'une imposture. En 1797, dans une lettre à James Madison, il déclara: «si une révolution commence en Angleterre, j'ai l'intention d'en être»(P. Foner 2:1395) ${ }^{3}$. Dans ses écrits, dont le style original, luimême révolutionnaire, frappe tous les commentateurs, il évoque à plusieurs reprises la révolution de Saint-Domingue, n'oublie ni l'Irlande ni l'Amérique du Sud, et compte sur Napoléon et ses batailles pour propager la révolution en Europe, notamment en Hollande. ${ }^{4}$ Ses intentions révolutionnaires ne se limitèrent pas à l'action politique, mais s'appliquèrent aussi au champ des croyances et des institutions religieuses. 
Dans la mesure où Paine était avant tout un pamphlétaire, c'est-à-dire un journaliste et un militant, sa vie fut inextricablement liée à l'expression de sa pensée révolutionnaire. Paine fut en outre un acteur des révolutions américaine et française au sens où il remplit des fonctions publiques - il fut quelque temps Secrétaire de la Commission des Affaires Étrangères aux États-Unis et siéga comme député français du Pas-de-Calais à la Convention. Aussi est-il inévitable d'en passer par sa biographie. ${ }^{5}$ On retracera donc d'abord la carrière de Thomas Paine. La cohérence de son projet révolutionnaire sera ensuite soulignée, projet qui s'articule autour de trois points centraux: le gouvernement, l'individu, la religion.

La révolution américaine, première révolution : Le sens commun et La crise américaine

Thomas Paine est né en 1737 dans le Norfolk dans une famille d'artisans obscure et plutôt pauvre. Il émigra en 1774, à l'âge de 37 ans, dans les colonies britanniques d'Amérique du Nord et s'installa à Philadelphie pourvu d'une lettre de recommandation de Benjamin Franklin, rencontré à Londres. À peine deux années plus tard, il fut propulsé sur le devant de la scène politique après qu'il eut exhorté, dans Le sens commun (Common Sense), les Américains à s'insurger contre l'Angleterre, à déclarer leur indépendance et à établir une république. Paine y mettait à mal toute tentative de réconciliation avec la couronne britannique, soulignait le comportement proprement monstrueux de la mère-patrie: "Je mets au défi l'avocat le plus ardent de la réconciliation de citer un seul avantage qui puisse revenir à ce continent du fait des liens avec la Grande-Bretagne ». Pour Paine, le moment était idéal, non seulement pour déclarer l'indépendance, mais aussi, puisque les colonies étaient jeunes et ne s'affrontaient pas encore, pour mettre en place un système républicain : «Le moment présent, c'est aussi ce moment particulier qui ne se présente pas deux fois à une même nation et où il lui est offert de se constituer en gouvernement ». L'occasion était donnée d'«entamer la constitution de notre gouvernement par le bon bout ", c'est-à-dire par la rédaction d'une charte. Paine voyait dans la révolution américaine le début d'une autre ère, «la naissance d'un monde nouveau », éclairé et républicain, et donnait une portée universelle aux événements (Le sens commun 99, 141, 163) : "L'Amérique ne s'est pas révoltée pour elle seule mais pour le monde entier » (Les droits de l'homme 151).

Publié en janvier 1776, Le sens commun, qui reprenait des idées qui circulaient depuis longtemps dans les colonies, connut un grand succès public (E. Foner 79). Premier bestseller américain, paru six mois avant l'adoption de la déclaration d'indépendance, ce "pamphlet hérétique " comme l'appela John Adams, fut loin d'être le seul pamphlet publié au cours de cette période agitée, mais aucun ne suscita autant d'opposition, d'enthousiasme et de débats passionnés (Keane 128). Cela à tel point qu'il est souvent considéré comme l'un des déclencheurs de la révolution américaine. Pour l'un des biographes de Paine, Le sens commun devint rapidement «le document politique de la première phase de la révolution » et « fit jaillir un nouvel esprit - jeune et audacieux, obsédé par le neuf, désireux d'expérimenter en concrétisant ce qui jusqu'à présent était jugé impensable ou irréaliste » (Keane 110). La crise américaine compléta Le sens commun. Cette série de 13 textes fut publiée entre 1776 et 1783, c'est-à-dire tout au long de la guerre d'indépendance. Dans le premier numéro, figure le célèbre "Voici venu, pour l'âme des hommes, le temps des épreuves» (Le sens commun 175). Paine y traite de la guerre et entend donner du courage aux patriotes. Dans le dernier numéro, il concluait avec confiance: "'Pour l'âme des hommes, le temps des épreuves' est fini. La 
révolution la plus grande et la plus parfaite que le monde ait jamais connue est glorieusement et heureusement achevée " (Le sens commun 195).

Les révolutions française et américaine, la révolution britannique à venir : Les droits de l'homme

Quelques années plus tard, c'est en plein cœur de la révolution française que se retrouva Paine. Il en avait proposé, d'Angleterre, une défense éloquente et célèbre, dans les deux parties des Droits de l'homme (Rights of Man) publiées en 1791 et 1792. Dans cet autre brûlot, Paine répondait aux attaques qu'avait formulées Edmund Burke dans sa Réflexion sur la révolution française (Reflection on the Revolution in France, 1790). Paine s'en prenait par la même occasion violemment à la monarchie britannique, notamment au système aristocratique et héréditaire, à « l'accouplement » de l'Église et de l'État, une "espèce de mulet qui ne sait que détruire et est incapable d'engendrer ", et à l'absence de constitution qui faisait du gouvernement britannique un gouvernement illégitime (Les droits de l'homme 80). Le but de Paine était de contribuer au mouvement radical républicain en Grande-Bretagne que les événements français avaient attisé et stimulé. Les droits de l'homme y eut une très forte influence - surtout la deuxième partie - et devint l'un des textes de référence du mouvement jacobin, mouvement rapidement condamné à la clandestinité sous l'effet de la répression (Thompson 114-121). Paine lui-même se retrouva en état d'arrestation suite à la proclamation royale contre les écrits séditieux de 1792.

7 Contraint par la justice du roi de fuir l'Angleterre et opportunément élu député du Pasde-Calais à la Convention (il avait été déclaré citoyen français par le décret révolutionnaire du 26 août 1792), Paine se retrouva précipité en France où il prit part, sans toutefois bien parler le français, aux débats révolutionnaires. Ses amitiés girondines et ses prises de position contre la mort du roi Louis XVI, en janvier 1793, mais aussi sa qualité d'Anglais, le rendirent suspect aux yeux des membres du Tribunal révolutionnaire et conduisirent à son enfermement dans la prison parisienne du Luxembourg à partir du 28 décembre 1793. Malgré la chute de Robespierre en juillet 1794, Paine ne fut libéré qu'ne novembre 1794. Faute d'une intervention diplomatique américaine efficace et en dépit du soutien de James Monroe, alors ministre américain en France, Paine était resté dix longs mois en prison, ce qui devait avoir pour effet une rancœur inextinguible à l'égard du président américain George Washington. Paine réintégra ensuite sa fonction de député à la Convention. Affaibli physiquement et politiquement, il ne fit qu'une apparition à l'assemblée, le 19 messidor an III (7 juillet 1795), lors des débats sur la nouvelle Constitution. Cette apparition unique fut toutefois remarquée: Paine s'y prononça contre le projet de constitution et pour le suffrage universel, position défendue dans sa Dissertation sur les premiers principes de gouvernement parue en France quelques mois auparavant.

La révolution religieuse : Le siècle de la raison

De prison, Paine avait commencé à rédiger Le siècle de la raison (The Age of Reason), qui fut publié en deux parties en 1794 et 1795. Pour lui, la révolution opérée dans le système politique, qui avait rendu à l'individu ses droits inaliénables et son autonomie, ne pouvait qu'être prolongée par une révolution religieuse. Dans le chapitre 1 de la première partie, il dit ainsi « Dès que j'eus publié en Amérique le pamphlet qui a pour titre le Sens Commun, je prévis qu'une révolution opérée dans le système de gouvernement serait très probablement suivie d'une autre révolution dans le système religieux ». Dans Le siècle de la raison, de façon particulièrement irrespectueuse, il 
s'attaquait au christianisme, dont il disait qu'il n'était "guère que l'idôlatrie des anciens mythologues adaptée aux exigences du pouvoir et du lucre». Dans ce pamphlet, Paine critiquait plus généralement toute religion révélée. Armé d'une hache, comme il le dit, il s'en prenait à la Bible, «tissu de mensonges, d'atrocités et de blasphèmes", et développait une apologétique déiste destinée à contrer "l'espèce d'athéisme " qu'était pour lui la religion chrétienne. Paine arguait que le déisme, religion rationnelle qui se limitait à la croyance en un Dieu unique et en une vie après la mort, et donc dénominateur commun à toutes les religions, finirait par triompher. En attendant, il affirmait dans sa "profession de foi »: "Ma propre conscience est ma seule église " (Le siècle de la raison 26, 29, 113, 56). Paine se faisait là le précurseur d'un type de religiosité très moderne, intériorisée, fondée sur la recherche personnelle et sur l'exploration spirituelle autonome de l'individu (Caron, 1999, 459).

Ce n'est qu'en 1802 que l'occasion lui fut donnée de retourner aux États-Unis. Le désir de voir proclamer en France une constitution selon ce qu'il jugeait être de vrais principes républicains - espoir déçu, la Convention avait rétabli le suffrage censitaire -, la perspective de voir une révolution éclater en Angleterre - désir lui aussi contrarié -, mais également la présence de navires anglais au large des côtes françaises - il était toujours en état d'arrestation en Angleterre - l'avaient jusque-là empêché de regagner ce qu'il considérait être son pays d'adoption. À son retour aux États-Unis, par ses «Lettres aux citoyens américains », Paine poursuivit la guerre des pamphlets qu'avait déclenchée la parution des Droits de l'homme et qui opposait les partisans de Jefferson, nouvellement élu président, et les fédéralistes, accusés de remettre en question les idéaux de la révolution (Conway $4: 381-429$ ). Toutefois, dans ces années, les espoirs de Paine portaient essentiellement sur la révolution religieuse qui selon lui était non seulement la conséquence logique de la révolution politique, mais dont il pensait aussi qu'elle pouvait rallumer la flamme de 1776. Alors que le clergé conservateur s'alarmait des idées radicales en provenance de la France et continuait de soutenir les fédéralistes, Paine s'investit dans des activités déistes et écrivit plusieurs textes destinés à poursuivre l'entreprise entamée avec Le siècle de la raison, qui avait d'ailleurs rencontré un vif succès dans la jeune République (la première partie du pamphlet fut rééditée 17 fois entre 1794 et 1796). En 1804, il écrivit pour Prospect ; or View of the Moral World, journal hebdomadaire déiste dirigé par l'un de ses coreligionnaires, Elihu Palmer, fondateur en 1794 de la première société déiste à New York.

10 Le progressisme révolutionnaire que Paine afficha simultanément dans les domaines politique, social et religieux lui valut un grand nombre d'ennemis et fit de lui une cible de choix. En décalage par rapport à son temps, Paine, provocateur, fut vilipendé, peu à peu marginalisé et finalement isolé. Paine avait pourtant été adulé lors de la publication du Sens commun et de La crise américaine à l'époque de la révolution américaine. Les droits de l'homme, quant à eux, avaient, aux début des années 1790, reçu un accueil passionné mais mitigé. Déclenchant une controverse féroce, par son rappel des idéaux de 1776, le pamphlet avait servi « de révélateur au débat latent sur le sens de la Révolution et de la République américaine » (Rossignol 118-119). Après la parution du Siècle de la raison, Paine fut non seulement diabolisé par ses adversaires qui firent de lui un athée, mais également abandonné par ses proches sur lesquels son radicalisme militant faisait de l'ombre - notamment Thomas Jefferson, qui cependant ne renia pas 
son amitié pour cet auteur contesté. Paine finit par mourir seul en 1809, après une longue maladie, à New York, dans un dénuement presque complet.

La révolution globale de l'homme nouveau

11 Dans le numéro XIII de La crise américaine, Paine notait : « Se sentir en mesure de rendre tout un continent heureux, enseigner à l'humanité l'art de l'être, présenter sur la scène de l'univers un personnage jusqu'alors inconnu et voir en quelque sorte une nouvelle création déposée entre nos mains, ce sont autant d'honneurs qui imposent la réflexion et qu'on saurait ni trop priser ni recevoir avec trop de gratitude " (Le sens commun 195-197). Il soulignait là, en 1783, la créativité du processus révolutionnaire. Le "personnage inconnu », c'est l'homme nouveau, ou plutôt réinventé, celui qui jusquelà sommeillait, " engourdi " par le principe héréditaire. Il est à présent indépendant, " fidèle à lui-même »; c'est un être qui peut penser de façon autonome et aspirer au bonheur, un être qui a recouvré ses droits inaliénables, droits naturels et droits civils, le vrai citoyen, à qui l'on donne des droits politiques, le droit de vote, le droit à la terre, le droit de vivre dignement. Le thème de l'homme nouveau est omniprésent dans Les droits de l'homme. Paine écrit ainsi : « La France n'a point égalisé, elle a exalté. Elle a fait en sorte que l'homme succède au nain». Le processus est irréversible et la contrerévolution impossible car: "Personne n'a encore découvert comment faire désapprendre à l'homme ce qu'il est ni comment faire en sorte qu'il n'ait point pensé ce qu'il a pensé » (Les droits de l'homme 171, 74, 112).

12 L'homme est au centre de la pensée révolutionnaire de Paine. Tout part de l'homme bien sûr, car seule l'action humaine rend l'acte révolutionnaire possible. L'homme de Paine est littéralement divinisé. Il devient à l'instar de Dieu, créateur. C'est un être véritablement colossal capable d'engendrer une révolution globale. Révolution globale plutôt qu'universelle, dans la mesure où malgré son progressisme, l'universalisme de Paine connaît des limites et son idéal « exlut au lieu d'inclure» (Thomson 79). Comme le remarque Elise Marienstras, « Même chez Paine, le plus 'universaliste' des penseurs révolutionnaires américains, l'universalisme n'est pas entier " (Marienstras 1992, 109; 1990, 62-63).

Certes Paine exprime sa solidarité avec l'humanité tout entière, et n'ignore ni l'Asie ni l'Afrique ${ }^{6}$. Ce n'est pas un philosophe abstrait, enfermé dans un bureau, c'est un homme du peuple, un homme de terrain, un militant qui prend des risques : il manifeste un attachement prononcé à un traitement concret des droits humains et, dans la deuxième partie des Droits de l'homme ou encore dans Justice agraire (1797) prône la mise en place d'un revenu minimum. Cependant, il met l'Europe et l'Amérique au cœur de sa réflexion et restreint son champ aux valeurs occidentales, ainsi qu'à la gent masculine et blanche. Il est, de ce point de vue, le symbole parfait des Lumières conquérantes. L'homme, qu'incarne le citoyen Paine et qui a remplacé Dieu au cœur du monde, refait le monde à son image et cette image n'est pas représentative de l'universalité du monde dans toute sa diversité sexuelle, ethnique et culturelle. Certains auteurs ont cherché à faire de Paine un champion des droits de tous; les femmes, cependant, sont largement exclues du projet révolutionnaire painien et les populations noires ou amérindiennes ne sont que rarement évoquées dans ses écrits publics. En 1776, Paine manifeste sa compassion pour le «malheureux Africain», mais il le fait dans une note (Conway $1: 154)$. Il mentionne assez fréquemment la situation à Saint-Domingue (dans sa correspondance privée avec Jefferson notamment), mais n'y consacre aucun écrit spécifique. La question rhétorique, « Do you want to renew in Louisiana the horrors of 
Domingo ? ", conclut une lettre condescendante aux Français esclavagistes du territoire de Louisiane, devenu américains, pour lesquels Paine recommande l'apprentissage de la langue française et l'adaptation de leurs pratiques culturelles catholiques, c'est-à-dire une forme d'acculturation (Conway $3: 436)^{7}$. Malgré des condamnations répétée de la traite des esclaves et de l'esclavage, il ne s'insurge jamais du fait que la constitution américaine, dont il fait l'éloge, pérennise l'esclavage. Comme le note Ann Thomson, «Les sentiments abolitionnistes de Paine ne donnent pas lieu à des publications, au contraire, il semble très réticent à toute expression publique de son opinion à ce sujet » (Thomson 66).

L'expression «révolution globale » renvoie plutôt à une méthodologie : la révolution politique, déclenchée par des individus éclairés et à laquelle Paine consacra la plus grande partie de sa carrière de propagandiste, est une étape dans un processus plus large. Autrement dit, à la façon des dominos qui posés côte à côte s'entraînent mutuellement lorsque le premier tombe sur le second, une révolution en appelle une autre. Il est très explicite sur ce point, notamment dans Les droits de l'homme. Voilà ce qu'il écrit en introduction à la deuxième partie: "Comme des révolutions ont commencé [...], on peut normalement s'attendre à d'autres révolutions.. [...] elles sont désormais un sujet universel de conversation et on peut dire d'elles qu'elles sont à l'ordre du jour [...]. ». En conclusion il observe :

A mesure que les réformes ou les révolutions, appelez-les comme bon vous semble, gagneront du terrain parmi les nations, celles-ci signeront entre elles des accords et des alliances et, quand plusieurs se seront ainsi confédérées, les progrès s'accélèreront jusqu'à l'extirpation totale du despotisme et de la corruption gouvernementale, du moins dans deux régions du monde, l'Europe et l'Amérique. On pourra alors ordonner aux barbaresques [the Algerine piracy] de cesser leur pirateries, car celles-ci ne doivent d'exister qu'aux rapports malveillants que les anciens gouvernements entretiennent entre eux (Les droits de l'homme 153, 268).

Conclusion

15 Paine est un eurocentriste américanophile, utopique et confiant. La révolution américaine a produit une onde de choc dont les effets se sont fait sentir en Europe, la révolution française doit rejaillir sur l'Angleterre ou l'Allemagne : «Le fer est chaud dans toute l'Europe » écrit-il en 1792 (Les droits de l'homme 266, 144). La révolution politique signifie la fin du despotisme et le recouvrement des droits humains inaliénables. S'ensuivent logiquement la naissance d'un nouvel individu, l'avènement d'une vraie liberté d'expression et d'une vraie liberté de conscience, la possibilité d'utiliser la raison sans contrainte, l'accès à l'éducation, la fin des injustices et de l'oppression des catégories exploitées ainsi que la paix internationale par le biais du commerce. Le soldat devient citoyen, le marin devient marchand, l'esclave devient libre. Quant à la révolution feminine, on peut émettre l'hypothèse que Paine comptait sur d'autres que lui, sur d'autres êtres éclairés tels que Mary Wollstonecraft qui, elle aussi, répondit à Burke et fit écho à Paine dans Défense des droits de la femme (Vindications of the Rights of Women, 1792).

16 La révolution religieuse, enfin, c'est-à-dire le retour à une idée pure de Dieu, parachève la révolution politique en lui permettant de perdurer. Dans Justice agraire, texte qui prône une forme de communisme, publié en 1797, Paine note que la révolution politique n'est pas suffisante en elle-même et doit être accompagnée de ce qu'il appelle une révolution de la civilisation ( « in the state of civilization »), que l'on peut comprendre comme désignant une révolution culturelle. Dans l'esprit optimiste de Paine, la 
révolution politique, dont il situe l'origine en Amérique, et l'accès à l'éducation qu'elle permet posent nécessairement et théoriquement les conditions d'une révolution des mœurs ainsi que d'une révolution religieuse. Pour lui, l'émergence d'un déisme commun, vecteur de progrès et de bonheur, devait finir par s'imposer, comme devait finir par s'imposer, sous l'égide des hommes blancs devenus libres, l'émancipation des esclaves et des femmes, comme celle de toutes les minorités opprimées. Paine était l'un de ces hommes éclairés qui devaient aider l'humanité à sortir de l'obscurité. La révolution globale est une œuvre collective, le fait d'une humanité retrouvée. Paine est une voix parmi d'autres. ${ }^{8}$

\section{BIBLIOGRAPHIE}

\section{Sources primaires}

BURKE, Edmund. Reflection on the Revolution in France. London, 1790.

CONWAY, Moncure Daniel, ed. The Writings of Thomas Paine, 4 vol. New York : AMS Press, 1967.

FONER, Philip S., ed. Complete Writings of Thomas Paine, 2 vol. New York : The Citadel Press, 1945.

PAINE, Thomas. Le sens commun-Common Sense, trad. Bernard Vincent. Paris : Aubier bilingue, 1983.

..... Le siècle de la raison. trad. rév. et prés. par Bernard Vincent. Nancy : Presses universitaires de Nancy, 1989.

.... Les droits de l'homme. trad. et prés. par Bernard Vincent. Nancy : Presses universitaire de Nancy ; Paris : Ligue des Droits de l'Homme, 1991.

WOLLSTONECRAFT, Mary. A Vindication of the Rights of Women. London, 1792.

La plupart des œuvres de Paine sont accessibles en ligne, voir http://www.thomaspaine.org/ contents.html

\section{Sources secondaires}

CARON, Nathalie. Thomas Paine contre l'imposture des prêtres. Paris : L'Harmattan, 1999.

.... « D'un continent à l'autre : Thomas Paine ou les déceptions d'un Américain ambitieux ». La France et les Amériques au temps de Jefferson et de Miranda. Dir. Marcel Dorigny et Marie-Jeanne Rossignol. Paris : Société d'études robespierristes, 2001. 31-45.

FONER, Eric. Tom Paine and Revolutionary America. New York : Oxford University Press, 1976.

JACOBY, Susan. Freethinkers : A History of American Secularism. New York : Metropolitan Books, 2004.

KAYE, Harvey J. Thomas Paine and the Promise of America. New York : Hill and Wang, 2005.

KEANE, John. Tom Paine: A Political Life. Londres : Bloomsbury, 1995.

LERAT, Christian, « Réactions américaines à une onde de choc: The Age of Reason de Thomas Paine (1737-1809) ». Sectes, Églises, Mystiques : échanges, conquêtes, métamorphoses. Dir. Bernadette RigalCellard. Bordeaux : Pleine Page éditeur, 2004. 317-334. 
MARIENSTRAS, Élise. Les mythes fondateurs de la nation américaine. Bruxelles : Complexe, 1992 (1976).

.... « L'universel et le particulier chez Thomas Paine ». Thomas Paine, citoyen du monde. Dir. George Kantin et Ligue des Droits de l'homme. Paris : Créaphys, 1990. 55-63.

ROSSIGNOL, Marie-Jeanne « Modernité de la république : Paine, Jefferson et l'impact de la révolution française en Amérique ». Thomas Paine et la République sans frontières. Dir. Bernard Vincent. Nancy : Presses universitaires de Nancy ; Paris : Ligue des droits de l'homme, 1993. 113-124.

THOMPSON, E.P. The Making of the English Working Class. Harmondsworth : Penguin, 1968 (1963). THOMSON, Ann. « Thomas Paine and the United Irishmen ». Études anglaises, juin $1991: 109-120$.

..... « Tom Paine et l'esclavage ». Nouveaux Regards sur l'Amérique. Peuples, nation, société. Perspectives comparatistes. Dir. Nathalie Caron et Naomi Wulf. Paris : Syllepse, 2004. 65-79.

VINCENT, Bernard, Thomas Paine ou la religion de la liberté. Paris : Aubier, 1987.

.... The Transatlantic Republican: Thomas Paine and the Age of Revolution. Amsterdam-New York, NY : Rodopi, 2005.

\section{NOTES}

1. Voir par exemple sa lettre à l'abbé Raynal à propos de la révolution américaine, « Letter addressed to the abbe Raynal on the affairs of North-America, in which the mistakes in the abbe's account of the revolution of America are corrected and cleared up », 1782 (Conway $2: 122$ ) : «Si l'originalité de la présente révolution est de faire naître un nouveau système, système où la civilisation sera étendue, elle recevra des cieux les preuves de l'approbation la plus forte; et dans la mesure où il s'agit là d'un sujet correspondant parfaitement bien aux compétences de l'abbé, je le lui recommande avec l'affection d'un ami et l'ardeur d'un citoyen universel » (traduction de l'auteur ; sauf mention contraire les traductions sont de l'auteur).

2. Dans Les droits de l'homme, Paine distingue entre révolutions passives (qui se produisent " pour éviter quelque grand désastre ou s'y soustraire ») et révolutions actives (" pour obtenir quelque grand bien positif »).

3. Lettre à James Madison, 27 avril 1797. Voir également Bernard Vincent 1987, 343. Selon B. Vincent, un document secret, de 1798, atteste que Paine devait faire partie du Directoire en Angleterre.

4. Voir par exemple Les droits de l'homme, 265 : «Il me semble également certain que les puissances confédérées mentionnées plus haut, de concert avec les États-Unis d'Amérique, pourraient efficacement proposer à l'Espagne l'indépendance de l'Amérique du Sud et l'ouverture de ces pays immenses et opulents au commerce général du monde, comme c'est déjà le cas pour l'Amérique du Nord ». Sur la révolution en Hollande suite aux succès militaires napoléoniens, voir la lettre à Samuel Adams, 6 mars 1795, et la lettre à un anonyme, 4 mars 1797, in P. Foner $2: 1376,1386$. Sur l'engagement de Paine en faveur des insurgés irlandais, voir Ann Thomson, 1991, 109-120. Concernant le style révolutionnaire de Paine, on peut se reporter à l'introduction du recueil d'articles de Bernard Vincent, 2005, chapitre intitulé « Storming the 'Bastille of Words' : Tom Paine's Revolution in Writing " (« La prise de la Bastille des mots : la révolution stylistique de Paine »). L'expression « Bastille de mots » 
vient de « Bastille d'un mot » utilisé par Paine lui-même à propos de l'utilisation des titres de noblesse qui emmurent l'individu : «Titles are like circles drawn by a magician's wand, to contract the sphere of man's felicity. He lives immured within the Bastille of a word » (Les droits de l'homme, 74 ; Conway $2: 320$ ).

5. Il existe plusieurs biographies de Paine. La plus récente est celle de John Keane. Voir aussi Bernard Vincent, 1987.

6. Des références sont faites à l'Asie et à l'Afrique dans Les droits de l'homme (152) par exemple : « Des progrès rapides accomplis en tous domaines par l'Amérique on peut rationnellement conclure que, si les gouvernements d'Asie, d'Afrique et d'Europe s'étaient appuyés à l'origine sur des principes semblables à ceux de l'Amérique, ou si la corruption ne les en avaient pas si tôt détournés, ces pays seraient à l'heure qu'il est dans un état supérieur à celui dans lequel ils se trouvent ". C'est moi qui souligne, l'affirmation étant teintée de préjugés.

7. "To the French Inhabitants of Louisiana », 22 septembre 1804. Le texte de Paine aux habitants de Louisiane, nous dit Bernard Vincent, contient « tous les pièges d'une leçon condescendante sur le civisme et les droits humains » (Vincent, 2005, 106). A propos d'une lettre à Thomas Jefferson, du 2 août 1803, Bernard Vincent ajoute « pour compléter son plan d'action, Paine proposa de défranciser la Louisiane par une instruction intensive » (105). En effet, dans cette lettre, Paine écrit au sujet des habitants de Louisiane, alors que le traité de cession ne date que de quelques mois (2 mai 1803) : « la langue dominante est aujourd'hui le français et l'espagnol, mais il sera nécessaire de mettre en place des écoles de façon à y enseigner l'anglais, car les lois seront rédigées dans la langue de l'Union. » (P. Foner 2 : 1441). Ce même jour, dans une lettre à son ami le sénateur Breckenridge, Paine note : « La religion établie [y] est la romaine ; cependant je ne sais pas ce qu'il en est des cérémonies extérieures (comme les processions ou les célébrations). Si [la Louisiane] avait continué d'être française, la religion, je suppose, aurait été mise sur le même plan que dans ce pays où aucune cérémonie religieuse ne peut se manifester sur les routes ou dans les rues. La même règle doit être établie ici, sinon il y aura des disputes entre les anciens colons et les nouveaux venus [...] ». La fin de cette phrase ainsi que la suite de la lettre montrent par ailleurs comment Paine envisageait la colonisation du nouveau territoire américain : «Les habitants actuels et leurs descendants seront en majorité pendant quelque temps, mais les nouveaux immigrants venus des anciens États et d'Europe, comme les mariages mixtes, changeront bientôt la situation telle qu'elle est aujourd'hui. C'est une chose à garder à l'esprit au moment où l'on prendra les premières mesures » (P. Foner 2 : 1446). 8. Après la mort de Paine, ses écrits ne restèrent pas lettre morte, loin s'en faut. Sa voix continua de se faire entendre et continue de se faire entendre. Au XIX ${ }^{\mathrm{e}}$ siècle, Les droits de l'homme devint le texte fondateur du mouvement ouvrier anglais (Thompson 2004, 99). De la même façon, Le siècle de la raison fut et demeure un texte clé de la libre pensée américaine, ce qu'illustre la parution récente de Freethinkers de Susan Jacoby qui fait la part belle à Thomas Paine (voir aussi Lerat 332). D'une manière générale, Paine est resté une référence, politique et religieuse. Utilisé aujourd'hui par la gauche américaine (voir par exemple le site web progressiste Tompaine.common sense (http:// www.tompaine.com/), il l'est aussi par les conservateurs (le président évangélique George W. Bush a ainsi cité l'auteur du Sens commun à plusieurs reprises). On assiste aujourd'hui à un regain d'intérêt pour les écrit de Thomas Paine, cela dans le contexte du débat sur les intentions des Père Fondateurs ("Founders Chic », appelle-t-on cette passion pour la période fondatrice de la nation américaine). Pour Harvey J. Kaye, auteur 
d'un livre au titre éloquent sur la persistance de Paine aujourd'hui, Thomas Paine and the Promise of America, " Paine est presque une célébrité ». Paine, dit-il, a bonne allure en comparison à d'autres Pères fondateurs : ce n'était pas un esclavagiste et il n'a pas cherché à s'enrichir personnellement $(9,13)$. Dans sa critique du livre de Kaye, l'historien Joseph Ellis remet en question la pertinence des références au XVIII ${ }^{\mathrm{e}}$ siècle pour agir dans le monde d'aujourd'hui : pour lui «transférer les idées et les mots de Paine dans notre monde, c'est comme planter des fleurs coupées » (« Thomas Paine and the Promise of America : Founding Father of the American Left » (New York Times, July 31 2005).

\section{AUTEUR}

NATHALIE CARON

Paris 10 